\title{
The effects of increased unilateral and bilateral calcaneal eversion on pelvic and trunk alignment in standing position
}

\author{
Jaehoon Yi \\ Division of Liberal Arts and Teaching, Sungkyul University, Anyang, Republic of Korea
}

Objective: Generally, it is known that there is a correlation between excessive calcaneus eversion and a patient with low back pain and it also affects pelvic alignment. However, there are not enough studies that show calcaneal eversion having an effect on the alignment of the trunk.

Design: Cross-sectional study.

Methods: A 3-dimensional motion analysis system was used to assess the lower limbs, pelvic alignment, and trunk alignment with increased unilateral and bilateral calcaneal eversion in twenty-one subjects. All subjects were asked to maintain a static posture for seven seconds on a wedge three times per posture for measurement and analysis purposes. The wedge used in the process was a lateral wedge with a 10-degree tilt to the lateral direction. To unify all of the subjects' foot position, the front and inner side of the wedge were marked. The height of the tilted wedge's inner side and flat wedge were balanced equally in order to be able to maintain the lateral part of the foot to the same height when producing an increased calcaneal eversion.

Results: Comparing the changes in trunk and pelvic alignment in accordance to calcaneal eversion for each posture, there was a significant different in the $\mathrm{X}$ and $\mathrm{Y}$-axis for each posture, but not in the $\mathrm{Z}$-axis $(p<0.05)$. Thus, it can be confirmed that calcaneal eversion in the sagittal plane and frontal plane may have and effect on the pelvis and the trunk.

Conclusions: Postures with increased bilateral and unilateral calcaneal eversion has an effect on pelvic alignment, but does not cause any changes in trunk alignment.

Key Words: Alignment, Calcaneal eversion, Pelvic, Trunk

\section{Introduction}

The role of the foot in standing position plays an important part as it provides the basis for supporting the weight and maintaining posture. The change of the frontal plane of the foot affects the alignment of the lower limbs. As a result, both pelvic and trunk are affected by the change $[1,2]$. Abnormal deformation of the foot causes an imbalance to the pelvis and the lower limbs which often become the cause of low back pain [3].

Previous studies show that subtalar joint pronation causes talus adduction, plantar flexion, and calcaneus eversion [4]. Talus adduction causes a functional reduction in length to in- duce abnormal alignment of the pelvic [5,6]. Also, bilateral presence of calcaneal eversion causes internal rotation of the hip joint, which increases pelvic anteversion and lumbar lordosis [1]. The increase of unilateral and bilateral calcaneal eversion causes abnormal alignment in the lower limbs which triggers pelvic lateral tilting and result in scoliosis [6]. The abnormal alignment in calcaneus eversion is deeply related with the occurrence of pathological conditions in the lumber spine and the cause of increasing low back pain $[5,7,8]$.

A recent study, which investigated the correlation of calcaneal eversion and the pelvis applying a wedge to the ankle, shows that anterior pelvic tilting increases when a wedge is

Received: 3 May, 2016 Revised: 2 June, 2016 Accepted: 7 June, 2016

Corresponding author: Jaehoon $\mathrm{Yi}$

Division of Liberal Arts and Teaching, Sungkyul University, 53 Sungkyuldaehak-ro, Manan-gu, Anyang 14097, Republic of Korea Tel: 82-31-467-8374 Fax: 82-31-449-0529 E-mail: eddyfox7@hotmail.com

(c) This is an Open-Access article distributed under the terms of the Creative Commons Attribution Non-Commercial License (http://creativecommons.org/licens es/by-nc/4.0) which permits unrestricted non-commercial use, distribution, and reproduction in any medium, provided the original work is properly cited.

Copyright $@ 2016$ Korean Academy of Physical Therapy Rehabilitation Science 
applied unilaterally or bilaterally. The torsion of the lateral pelvis increased when a wedge was applied unilaterally [9], and when a wedge was applied in the angle of 5 and 10 degrees, the unilateral calcaneal eversion increase had an effect in increasing hip, pelvic and thoracic angle irrespectively [10]. Moreover, the pressure variation of low-arch and high-arch of the foot and pelvic alignment, which tested plantar pressure distribution between adduction and abduction, differs according to the height of the arch [11].

However, previous research focuses on finding out the fact of how calcaneal eversion affects the lower limbs and pelvic body alignment. It is insufficient to demonstrate the effects of calcaneal eversion causing some kind of change in the pelvis, trunk, and the whole body. This study focuses on not only the relationship between the calcaneus and the pelvic but also the overall linkage of the calcaneus, pelvis, and trunk through analyzing the changes in the trunk. Furthermore, we suggest the trends and direction of wedge use by offering Z-axis change and rotation data. To this end, the change in trunk and pelvis caused by unilateral or bilateral calcaneus is analyzed with the help of a 3-dimensional (3D) motion analysis system.

\section{Methods}

\section{Subjects}

Eleven healthy male (age, $30.4 \pm 4.34$ year; height, $172.4 \pm$ $6.31 \mathrm{~cm}$; weight, $70.5 \pm 8.14 \mathrm{~kg}$ ) and 10 female adults (age, $28.2 \pm 5.49$ year; height, $163.3 \pm 4.60 \mathrm{~cm}$; weight, $54.0 \pm 5.75$ $\mathrm{kg}$ ) with no recent orthopedic history in six months participated in the study. Also, prior to the experiment, the study purpose, importance and procedure were fully explained to the subject. The experiment was conducted after receiving a participation agreement from each participant.

\section{Assessment tools and experimental methods}

In order to collect kinematic data, the 6 Oqus 100 infrared camera produced by 3D motion analysis system (Qualisys, Göteborg, Sweden, 2011) was used to photograph the changes in lower limb, pelvic, and trunk alignment. Sampling rate was set up at 150 frames/sec to track the position of the reflection markers attached to the whole body which made it possible to obtain 3D location coordinates in real-time. The diameter of the reflection markers of the Oqus 100 camera that was used in infrared tracking was $19 \mathrm{~mm}$ and calibration markers were attached to the anatomical boundary of the body segments to measure the subjects' anatomical body alignment states. The markers for measuring movement which was designed to minimize the margin of error was equipped with a fixed $19 \mathrm{~mm}$ reflection marker cluster [12-14].

Standing position in a relaxed state (control condition), standing position utilizing a unilateral wedge (unilateral condition) that was made of wood $(30 \times 15 \times 2 \mathrm{~mm})$, and standing position utilizing bilateral wedge (bilateral condition) were the three measuring positions. Thirty-five markers were used for this experiment. The wedge used in the process was a lateral wedge with a 10-degree tilt to the lateral direction. To unify all subjects' foot position, the wedge's front and inner side were marked. The height of the tilted wedge's inner side and flat wedge were balanced equally. This was to maintain the lateral part of the foot in the same height when increasing calcaneus eversion.

Experimental procedure in accordance with the wedge type was carried out randomly and the change was given to the right lower limbs only in order to normalize unilateral arbitration. All subjects were asked to maintain a static posture for 7 seconds on the wedge repeated three times per each posture to be measured and analyzed (Figure 1).

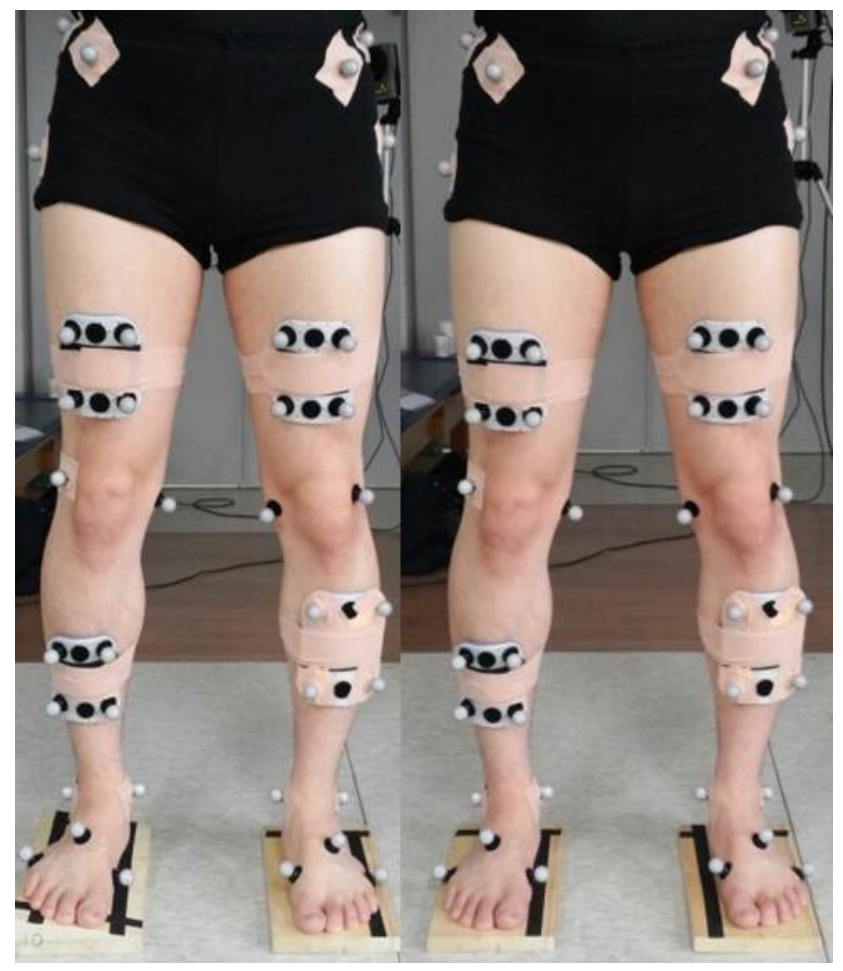

Figure 1. The wedge used in the experiments. 
Table 1. Comparison of trunk and pelvic change among three conditions $(\mathrm{N}=21)$

\begin{tabular}{|c|c|c|c|c|}
\hline Variable & Control condition & Unilateral & Bilateral & $\mathrm{F}$ \\
\hline Trunk $\mathrm{X}$-axis & $-12.95(2.79)$ & $-13.25(2.78)$ & $-13.39(2.55)$ & $595.628^{*}$ \\
\hline Trunk Y-axis & $1.15(1.99)$ & $1.11(1.78)$ & $1.18(2.15)$ & $7.028^{*}$ \\
\hline Trunk Z-axis & $1.70(9.32)$ & $2.16(9.69)$ & $1.81(9.03)$ & 0.745 \\
\hline Pelvic $\mathrm{X}$-axis & $3.2(6.39)$ & $3.26(7.4)$ & $2.82(6.85)$ & $5.465^{*}$ \\
\hline Pelvic Y-axis & $0.42(1.59)$ & $3.74(1.96)$ & $0.72(1.94)$ & $19.297^{*, \mathrm{a}}$ \\
\hline Pelvic Z-axis & $0.33(2.78)$ & $0.11(3.64)$ & $0.21(3.81)$ & 0.174 \\
\hline Right ankle Y-axis & $11.56(5.05)$ & $15.93(4.95)$ & $15.46(4.89)$ & $187.045^{*}$,a \\
\hline Left ankle Y-axis & $-11.18(5.82)$ & $-8.78(5.19)$ & $-14.69(5.75)$ & $67.293^{*, \mathrm{a}}$ \\
\hline
\end{tabular}

Values are presented as mean (SD). Positive values: pelvic anteversion, pelvic tilt for the right side, calcaneal eversion. Negative values: pelvic retroversion pelvic tilt for the left side, calcaneal inversion.

${ }^{*} p<0.05$. ${ }^{\mathrm{a}}$ Statistical difference between unilateral and bilateral $(p<0.05)$.

\section{Data analysis}

Qualisys Track Manager ver. 2.7 program was used to obtain the position coordinates. Raw data obtained in the process was filtered with Second order Butterworth Bidirectional Filter to eliminate the margin of error caused by noise and cut-off frequency was set at $6 \mathrm{~Hz}$. Visual 3D program (V4.96; C-Motion Inc., Germantown, MD, USA) was utilized for modeling body segment and calculating variables. Repeated ANOVA was carried out for Kinematic data, which was calculated through Visual 3D program, utilizing PASW Statistics ver. 18.0 (IBM Co., Armonk, NY, USA) statistics package to verify statistical difference between each group. Statistical significance level was set at $\alpha=0.05$.

\section{Results}

The posture change of the pelvis and trunk in accordance with calcaneus eversion

There was a significant difference in the $\mathrm{X}$-axis (flexion \& extension), Y-axis (abduction \& adduction) comparing trunk and pelvic change in accordance with calcaneus eversion for each posture $(p<0.05)$ but there was no significant difference in Z-axis (rotation; Table 1). In other words, calcaneus eversion only affected pelvic and trunk angle in the sagittal and frontal planes.

\section{Trunk posture}

Standing position for unilateral and bilateral wedge in sagittal plane did not show a significant difference in trunk posture compared with the standing position in a relaxed state (controlled position). The mean difference in rotation angle change was $-0.28(\mathrm{SD}, 0.36$; $\mathrm{CI},-1.018-0.465$; $p=0.45),-0.46$ (SD, 0.37; CI, $-1.221-0.310 ; p=0.23$ ). Unilateral and bilateral wedge posture did not show a significant difference in trunk posture compared with the controlled position. The calculated mean difference of lateral bending was $-0.08^{\circ}$ (SD, 0.21; CI, - 0.528-0.361; $p=0.70),-0.01^{\circ}$ (SD, 0.27; CI, $\left.-0.582-0.574 ; p=0.99\right)$.

\section{Pelvic posture}

The posture with unilateral and bilateral wedge did not show a significant difference for the trunk posture in the sagittal plane when compared with the controlled position. The mean difference of the rotation angle change was $0.17^{\circ}(\mathrm{SD}$, 0.48 ; CI, $-0.833-1.175 ; p=0.73),-0.28^{\circ}$ (SD, 0.38 ; CI, $1.074-0.523 ; p=0.16)$. However, in the unilateral and bilateral wedge position, there was a significant difference in trunk posture compared with controlled position in the frontal plane. The mean difference of lateral tilting was measured to be $3.30^{\circ}(\mathrm{SD}, 0.21 ; \mathrm{CI}, 2.856-3.740 ; p=0.00), 0.30^{\circ}$ (SD, 0.19; CI, $-0.094-0.702 ; p=0.13$ ).

\section{Discussion}

In the case of calcaneal eversion, an increase in anterior and bilateral pelvic posture which has been clearly proven by Pinto et al. [9]. They observed $1.57^{\circ}$ average anterior transposition and $7.5^{\circ}$ to the right and $8.36^{\circ}$ to the left of average calcaneal eversion [1]. Our study tries to prove the variables of pelvic change in accordance with calcaneal eversion including the trunk's three-dimensional direction and the transverse plane of the pelvis which was not included in Pinto et al. [9]. When using a wedge $10^{\circ}$ tilted inward due to bilateral tendency, we discovered that there is an average of pelvic anterior $0.4^{\circ}$ and calcaneus eversion $3.5^{\circ}-4^{\circ}$. 
The pelvis went through the transposition to anterior in accordance with calcaneal eversion and there was an increase in bilateral tendency. The previous research from Pinto et al. [9], Khamis and Yizhar [1] shows the same result with this study concerning calcaneal eversion and lower limbs [1,9]. However, the increase of bilateral tendency and pelvic anterior transposition is rather low in our study compared with the previous researches. Such difference can be the result of measuring the figure with the assumption of the foot being a single segment, as a result, the movement of the calcaneus could have been underestimated. According to the research by Tateuchi et al. [10], pelvic anterior transposition was $1.57^{\circ}$ in weight supported unilateral condition showing the fact that there are relatively individual differences.

Previous studies utilizing wedge analyzed the calcaneal position in $\mathrm{X}$ and $\mathrm{Y}$ axes but our study analyzed it in three directions. This is due to the fact that we considered three directional analysis as the movement of the calcaneus in the ankle joint complex occurs in three side of the oblique axis [4]. While previous studies show that the directional changes of the calcaneus are larger in the bilateral condition than the unilateral condition [1,9], our study shows that the center of gravity moves toward the inner side further in the unilateral condition by measuring the result value in a threedimensional measurement. But in the bilateral condition, the center of gravity moving toward the center decreases because of compensatory action in the bilateral condition compared to the unilateral condition.

In our study, we observed that there is a significant change since when calcaneus calcaneus is unilateral, pelvic anterversion is $0.4^{\circ}$. This result shows that calcaneal eversion which increases asymmetrically causes a significant change in pelvic posture in the sagittal plane.

There is an interdependent mechanism between calcaneal eversion and pelvic anteversion. This happens along with subtalar pronation and calcaneal eversion. It generates the lower limb's internal rotation in the hip joint $[1,8]$, positions the femoral head backward, and induces posterior movement of the pelvis. This kind of posture modification is a method to maintain the balance of the body through moving the trunk forward and it can be accomplished by pelvic anteversion [9].

Lateral pelvic tilt is derived by the increase in calcaneal eversion which consequently shortens the ipsilateral lower limb when using a unilateral wedge $[6,8]$. Therefore, excessive asymmetric calcaneal eversion can cause lateral tilt of the sacral base which results in induced lumbar scoliosis
$[6,7]$.

The observed variable value increase in calcaneal eversion can be regarded as the result of using a wedge which has medial inclination and having an effect in ankle joint complex posture in the standing position. These results can be confirmed through the clinical field [9].

The eversion angle increase in the right calcaneus is equivalent to the angle variation of the pelvis and this change affects the posture of lower limbs through interaction forces between segments [15].

The lumbar posture is dependent on the pelvic alignment in standing position. As a result, pelvic anteversion \& lateral tilt can cause lumbar hyperlordosis and scoliosis [6]. Lumbar hyperlordosis is the result of increasing load to the articular surfaces and related with low back pain [16,17]. Therefore, the pelvic alignment change in our study can be the cause of lower back pain. Levine and Whittle [2] said that pelvic anteversion increases lumbar lordosis by $10.8^{\circ}$ and observed that lumbar lordosis increased by 7 degrees in patients who suffered from back pain [3]. According to Legaye et al. [18], $1^{\circ}$ pelvic anteversion change causes $1^{\circ}$ lumbar lordosis in linear relation of the pelvis and lumbar in the sagittal plane position. This kind of change in the spine can be a risk factor of low back pain [19-22]. It is clinically appropriate to see that the cause of low back pain which results from pelvic anterolisthesis is due to the reduced tension of abdominal muscles, hip extensor muscles and increased tension of hip flexor muscles [23].

The relationship of calcaneal eversion and trunk angle change is a phenomenon that occurs when subjects try to recover their balance after the pelvis is tilted forward while the calcaneus is everted $10^{\circ}$. In the case of malalignment syndrome of the calcaneus, it is reported that pelvic tilting that occurs in the sagittal plane and frontal plane is one of the main causes [10].

Calcaneal eversion changes the trunk and pelvic segment angle by lateral tilting. The trunk segment lateral rotation in the frontal plane showed significant increase in the calcaneal eversion condition but pelvic lateral rotation was not observed.

Trunk lateral rotation increase is not a change resulting from increased rotation momentum but a phenomenon that occurs when the tilted upper body and pelvic lateral tilting is combined in the standing position. Though, trunk rotation does not occur in accordance with pelvic rotation, rotation of the spine, which is a cause of calcaneal eversion, increases between the trunk and the pelvis. Consequently, the human body adapted to unbalanced posture can change the body 
posture that can cause greater disease in the future [24].

Although generating calcaneal eversion through the utilization of a wedge does not effect pelvic segment change, it affects the three-dimensional kinematic angle in the trunk. The pelvic eversion in the bilateral and unilateral group which was generated by calcaneal eversion caused small but meaningful changes in pelvic alignment and is the cause of increased anterior pelvic tilt. Also, unilateral eversion posture can induce outer pelvic tilt. Bilateral and unilateral eversion do not show significant changes in trunk alignment.

The results in various researches appear the same regardless of different application method of a wedge. So, it can be presumed that wedge angle change affects pelvic angle change. Thus, our study is helpful in demonstrating the effect of calcaneal angle change related to the pelvis since we offer more precise figures through three-dimensional measurements. In future studies, it is necessary to demonstrate the correlation of the trunk and the pelvic through experiments concerning various directions and confirm the possibility of related diseases progression.

\section{Conflict of Interest}

The authors declared no potential conflicts of interest with respect to the authorship and/or publication of this article.

\section{References}

1. Khamis S, Yizhar Z. Effect of feet hyperpronation on pelvic alignment in a standing position. Gait Posture 2007;25:127-34.

2. Levine D, Whittle MW. The effects of pelvic movement on lumbar lordosis in the standing position. J Orthop Sports Phys Ther 1996;24:130-5.

3. Christie HJ, Kumar S, Warren SA. Postural aberrations in low back pain. Arch Phys Med Rehabil 1995;76:218-24.

4. Rockar PA Jr. The subtalar joint: anatomy and joint motion. J Orthop Sports Phys Ther 1995;21:361-72.

5. Rothbart BA, Estabrook L. Excessive pronation: a major biomechanical determinant in the development of chondromalacia and pelvic lists. J Manipulative Physiol Ther 1988;11:373-9.

6. Gurney B. Leg length discrepancy. Gait Posture 2002;15:195206.

7. Aebi M. The adult scoliosis. Eur Spine J 2005;14:925-48.

8. Botte RR. An interpretation of the pronation syndrome and foot types of patients with low back pain. J Am Podiatry Assoc 1981;
71:243-53.

9. Pinto RZ, Souza TR, Trede RG, Kirkwood RN, Figueiredo EM, Fonseca ST. Bilateral and unilateral increases in calcaneal eversion affect pelvic alignment in standing position. Man Ther 2008;13:513-9.

10. Tateuchi H, Wada O, Ichihashi N. Effects of calcaneal eversion on three-dimensional kinematics of the hip, pelvis and thorax in unilateral weight bearing. Hum Mov Sci 2011;30:566-73.

11. Wong L, Hunt A, Burns J, Crosbie J. Effect of foot morphology on center-of-pressure excursion during barefoot walking. J Am Podiatr Med Assoc 2008;98:112-7.

12. Karlsson D, Tranberg R. On skin movement artifact-resonant frequencies of skin markers attached to the leg. Hum Mov Sci 1999:18:627-35.

13. Fuller J, Lui LJ, Murphy MC, Mann RW. A comparison of lower-extremity skeletal kinematics measured using skin- and pin-mounted markers. Hum Mov Sci 1997;16:219-42.

14. Reinschmidt C, Van den Bogert AJ, Nigg BM, Lundberg A, Murphy N. Effect of skin movement on the analysis of skeletal knee joint motion during running. J Biomech 1997;30:729-32.

15. Zajac FE, Neptune RR, Kautz SA. Biomechanics and muscle coordination of human walking. Part I: introduction to concepts, power transfer, dynamics and simulations. Gait Posture 2002;16: 215-32.

16. Shirazi-Adl A, Drouin G. Load-bearing role of facets in a lumbar segment under sagittal plane loadings. J Biomech 1987;20:601-13.

17. Steinberg EL, Luger E, Arbel R, Menachem A, Dekel S. A comparative roentgenographic analysis of the lumbar spine in male army recruits with and without lower back pain. Clin Radiol 2003;58:985-9.

18. Legaye J, Duval-Beaupère G, Hecquet J, Marty C. Pelvic incidence: a fundamental pelvic parameter for three-dimensional regulation of spinal sagittal curves. Eur Spine J 1998;7:99-103.

19. Duncan NA, Ahmed AM. The role of axial rotation in the etiology of unilateral disc prolapse. An experimental and finite-element analysis. Spine (Phila Pa 1976) 1991;16:1089-98.

20. Gordon SJ, Yang KH, Mayer PJ, Mace AH Jr, Kish VL, Radin EL. Mechanism of disc rupture. A preliminary report. Spine (Phila Pa 1976) 1991;16:450-6.

21. Manning DP, Mitchell RG, Blanchfield LP. Body movements and events contributing to accidental and nonaccidental back injuries. Spine (Phila Pa 1976) 1984;9:734-9.

22. Marras WS, Granata KP. A biomechanical assessment and model of axial twisting in the thoracolumbar spine. Spine (Phila $\mathrm{Pa}$ 1976) 1995;20:1440-51.

23. Harris-Hayes M, Sahrmann SA, Norton BJ, Salsich GB. Diagnosis and management of a patient with knee pain using the movement system impairment classification system. J Orthop Sports Phys Ther 2008;38:203-13.

24. Mueller MJ, Maluf KS. Tissue adaptation to physical stress: a proposed "Physical Stress Theory" to guide physical therapist practice, education, and research. Phys Ther 2002;82:383-403. 